INPLASY

PROTOCOL

To cite: Guo et al. The efficacy and safety of topical tranexamic acid for spinal surgery: a meta-analysis. Inplasy protocol 202160028. doi:

10.37766/inplasy2021.6.0028

Received: 09 June 2021

Published: 09 June 2021

Corresponding author: Junjie Wang

piccolovegeta@sina.com

Author Affiliation:

Qingdao University

Support: None.

Review Stage at time of this submission: The review has been conducted, submitted and revised.

Conflicts of interest:

None declared.

\section{The efficacy and safety of topical tranexamic acid for spinal surgery: a meta-analysis}

Guo, T1; Xuan, W2; Feng, H33 Wang, J4; Ma, X5.

Review question / Objective: Our review evaluated the effect of topical tranexamic acid on blood loss during and after spinal surgery via a comprehensive meta-analysis of the published data in RCTs and other comparative cohort studies. Condition being studied: Spinal surgeries are often accompanied by significant blood loss both intraoperatively and postoperatively. Excessive blood loss caused by surgery may lead to several unsatisfactory medical consequences. Tranexamic acid (TXA) is a kind of antifibrinolytic agent that has been widely used in spinal surgery. Currently, it is widely accepted that intravenous TXA (ivTXA) can clearly reduce blood loss in spinal fusion surgeries. Compared with ivTXA, topical TXA (tTXA) seems to be much easier to administer, and this advantage provides a maximum concentration of TXA at the haemorrhagic site with little to no TXA entering the circulation.

INPLASY registration number: This protocol was registered with the International Platform of Registered Systematic Review and Meta-Analysis Protocols (INPLASY) on 09 June 2021 and was last updated on 09 June 2021 (registration number INPLASY202160028).

\section{INTRODUCTION}

Review question / Objective: Our review evaluated the effect of topical tranexamic acid on blood loss during and after spinal surgery via a comprehensive meta-analysis of the published data in RCTs and other comparative cohort studies.
Condition being studied: Spinal surgeries are often accompanied by significant blood loss both intraoperatively and postoperatively. Excessive blood loss caused by surgery may lead to several unsatisfactory medical consequences. Tranexamic acid (TXA) is a kind of antifibrinolytic agent that has been widely used in spinal surgery. Currently, it is widely 
accepted that intravenous TXA (ivTXA) can clearly reduce blood loss in spinal fusion surgeries. Compared with ivTXA, topical TXA (tTXA) seems to be much easier to administer, and this advantage provides a maximum concentration of TXA at the haemorrhagic site with little to no TXA entering the circulation.

\section{METHODS}

Search strategy: Two trained researchers independently searched major online databases, including PubMed, EMBASE, the Web of Science, and the Cochrane Central Register of Controlled Trials, on October 21, 2020. Different combinations of the following terms were used to perform the search: "interbody fusion", "lumbar fusion", "spinal fusion", "spinal instrument", "spinal surgery", "spine", "I umbar degenerative disease", "tranexamic acid", and "TXA".

Participant or population: Patients who underwent any type of spinal surgery.

Intervention: Any tTXA treatment was administered to the experimental group, and a placebo or standard treatment was administered to the control group.

Comparator: Parameters that described blood loss volume and surgical effects, including drainage volume, blood loss volume, serum haemoglobin level, length of hospital stay, side effects and complications.

Study designs to be included: The type of study was an RCT or comparative cohort study.

Eligibility criteria: (1) the participants were patients who underwent any type of spinal surgery; (2) the intervention, including any tTXA treatment, was administered to the experimental group, and a placebo or standard treatment was administered to the control group; (3) the outcomes were parameters that described blood loss volume and surgical effects, including drainage volume, blood loss volume, serum haemoglobin (HB) level, length of hospital stay, side effects and complications; (4) the type of study was an RCT or comparative cohort study; and (5) the study was published in English.

Information sources: PubMed, EMBASE, the Web of Science, and the Cochrane Central Register of Controlled Trials.

Main outcome(s): Total blood loss, hidden blood loss, intraoperative blood loss, total postoperative drainage volume, drainage tube duration postoperatively, drainage volume and drainage of blood content at postoperative day (POD) 1 and POD2, length of hospital stay, number of patients who received a blood transfusion, serum haemoglobin level at POD1, operative timespan, side effects and complications.

Quality assessment / Risk of bias analysis: The Cochrane risk of bias tool was used to evaluate the methodological quality of the RCTs, while the Newcastle-Ottawa Scale was used to assess that of the retrospective or prospective cohort studies.

Strategy of data synthesis: The metaanalysis was performed using RevMan 5.3. Using a random-effects model, odds ratios (ORs) and $95 \%$ confidence intervals (Cls) were expressed for the dichotomous variables. Using a fixed-effects model, the mean difference (MD) and $95 \% \mathrm{Cls}$ were calculated for the continuous outcomes. Statistical significance was deemed to exist when $P<0.05$. The heterogeneity across studies in the comparisons was identified using the chi-square $(x 2)$ and 12 tests; if $p 50 \%$, heterogeneity was considered to exist. For the continuous outcomes, if $\mathrm{p} 50 \%$, a random-effects model was applied.

Subgroup analysis: We did not perform subgroup analysis.

Sensitivity analysis: We did not perform sensitivity analysis.

Country(ies) involved: China. 
Keywords: spinal surgery; topical tranexamic acid; blood loss; rehabilitation; efficacy and safety; meta-analysis; randomized controlled trials.

Contributions of each author:

Author 1 - Tai Guo.

Email: 466361889@qq.com

Author 2 - Wenxia Xuan.

Email: 329795990@qq.com

Author 3 - Haoyu Feng.

Email: 838669221@qq.com

Author 4 - Junjie Wang.

Email: piccolovegeta@sina.com

Author 5 - Xun Ma.

Email: maxun2532@sina.com 